\title{
Model Fusion Using Fuzzy Aggregation: Special Applications to Metal Properties
}

\author{
Qian Zhang ${ }^{1}$, Mahdi Mahfouf ${ }^{1}$, John R. Yates ${ }^{2}$, and Christophe Pinna ${ }^{3}$ \\ ${ }^{1}$ Department of Automatic Control and Systems Engineering, \\ The University of Sheffield, Sheffield, S1 3JD, UK \\ (E-mail addresses: qian.zhang@sheffield.ac.uk; m.mahfouf@sheffield.ac.uk) \\ ${ }^{2}$ School of Mechanical, Aerospace and Civil Engineering, \\ The University of Manchester, M60 1QD, UK \\ ${ }^{3}$ Department of Mechanical Engineering, \\ The University of Sheffield, Sheffield, S1 3JD, UK
}

\begin{abstract}
To improve the modelling performance, one should either propose a new modelling methodology or make the best of existing models. In this paper, the study is concentrated on the latter solution, where a structure-free modelling paradigm is proposed. It does not rely on a fixed structure and can combine various modelling techniques in 'symbiosis' using a 'master fuzzy system'. This approach is shown to be able to include the advantages of different modelling techniques altogether by requiring less training and by minimising the efforts relating optimisation of the final structure. The proposed approach is then successfully applied to the industrial problems of predicting machining induced residual stresses for aerospace alloy components as well as modelling the mechanical properties of heat-treated alloy steels, both representing complex, non-linear and multi-dimensional environments.
\end{abstract}

Keywords: data-driven modelling; fuzzy system; model fusion; engineering material; residual stress; mechanical property; aluminium alloy; steel

\section{Introduction}

For describing various physical and social systems in nature, different models and their associated modelling methodologies have been developed. In order to improve the performance of a model, there are two general strategies. The first strategy is to develop an entirely new modelling paradigm considering the specific features of an object system, which are not coped with by 'old' methods. The second strategy relates to making the best of existing modelling paradigms. This is usually achieved via two ways: further optimisation or systematic combination with other types of models.

In this paper, the research study is focused on the idea of a systematic combination of various models. In reality, one single modelling paradigm cannot always perform well 
due to the different components, or different characteristics of a complex system under different situations. In this case, the combination of various models may cover a wider range of model formulations and provides a more flexible modelling structure. However, when given a modelling problem without any preliminary knowledge about it, it is very difficult to choose the most appropriate modelling approach. In such a situation, the systematic combination strategy will automatically find a suitable structure, whereby adequate modelling methods will be fully employed while inadequate ones will be adjusted to affect the few.

To achieve a sophisticated combination of different types of models, a linear combination is far from enough. In this paper, a fuzzy rule-based system [1, 2] is designed as a high-level master system to handle the cooperation of low-level submodels, since fuzzy systems are inherently non-linear topologies which are known to be universal approximators [3] and can deal with the curse of dimensionality effectively $[4,5]$.

The following sections in the paper are organised as follows. Section 2 introduces the proposed modelling paradigm in details. In Section 3, the new proposed approach is validated using two benchmark problems, one is a problem of static non-linear system approximation and the other is a dynamic system identification problem. In Section 4, it is further applied to two industrial problems, which aim at modelling machining induced residual stresses in aluminium alloy components and the prediction of mechanical properties of alloy steels. Finally, Section 5 concludes this paper.

\section{The Proposed Paradigm for Combining Various Modelling Methodologies}

\subsection{Introduction to fuzzy systems}

Fuzzy rule-based systems [1, 2] are viewed as robust 'universal approximators' capable of performing non-linear mappings between inputs and outputs. It is an approach that allows a system to be represented using a descriptive language (linguistic 'IF-THEN' rules) [6], which can easily be understood and explained by humans in order for them to gain a deeper insight into more often than not uncertain, complex, and ill-defined systems.

Generally, a fuzzy system consists of four fundamental components: fuzzifiers, a fuzzy rule-base, a fuzzy inference engine, and defuzzifiers. The central part of a fuzzy system is the knowledge-base (rule-base) consisting of fuzzy rules. A fuzzy rule is an IF-THEN statement in which some words are characterised by continuous membership functions. Specifically, a fuzzy rule-base comprises the following fuzzy rules:

$$
\text { Rule }_{l} \text { IF } x_{1} \text { is } A_{1}^{l} \text { AND ... AND } x_{i} \text { is } A_{i}^{l} \text {, THEN } y \text { is } B^{l} \text {, }
$$

where $l=1,2, \ldots, N_{c} ; N_{c}$ is the number of rules in the fuzzy rule-base; $A_{i}^{l}$ and $B^{l}$ are 
fuzzy sets in $U_{i} \subset R$ and $V \subset R$, respectively, and $\boldsymbol{x}=\left[x_{1} x_{2} \ldots x_{i}\right]^{T} \in U$ and $y \in V$ are the input and output (linguistic) variables of the fuzzy system, respectively.

The fuzzifier is defined as a mapping from a real-valued point $x^{*} \in U \subset R^{n}$ to a fuzzy set $A^{*}$ in $U$. In a fuzzy inference engine, fuzzy logic principles direct how to employ the fuzzy rules into a mapping from an input fuzzy set $A^{*}$ to an output fuzzy set $B^{*}$. The defuzzifier is a mapping from the output fuzzy set $B^{*}$ in $V \subset R$ to a real-valued point $y^{*} \in V$. Conceptually, the purpose of the defuzzifier is to specify a point in $V$ that best represents $B^{*}[3]$.

Generally, when constructing a fuzzy system, the fuzzy inference engine and the defuzzifiers are predefined. Thus, the primary work will be centred around the idea of generating appropriate fuzzifiers (fuzzy sets) and an appropriate fuzzy rule-base.

\subsection{Master fuzzy systems}

To handle the combination of different modelling techniques, a singleton fuzzy system [7], which is referred to as 'master fuzzy system', is designed. As shown in Fig. 1, the proposed architecture includes a range of pre-developed sub-models, a master fuzzy rule-base, a fuzzy inference engine. A master fuzzy rule is employed to 'decide' which sub-model should be activated in a certain circumstance. When a set of inputs are imported to the master fuzzy system, the fuzzy inference engine will process the given inputs and the activated sub-models to produce a final combined output.

\section{$<$ Figure 1 $>$}

The general form of the master fuzzy rules is as follows:

Rule $R_{n}$ : IF $x_{1}$ is $A_{1}{ }^{n}$ AND ... AND $x_{m}$ is $A_{m}{ }^{n}$, THEN $y$ is $Y^{n}\left(Y_{F M}, Y_{A N N}, Y_{L R}\right.$, etc.),

where $R_{n}$ is the label of the $n$th fuzzy rule; $\boldsymbol{x}=\left[\begin{array}{llll}x_{1} & x_{2} & \ldots & x_{m}\end{array}\right]^{\mathrm{T}} \in U_{1} \times U_{2} \times \ldots \times U_{m}$ are input linguistic variables, $m$ is the number of inputs; $A_{i}^{n}$ are the antecedent fuzzy sets of the universes of discourse $U_{i}$, where $i=1,2, \ldots, m ; y \in V$ is the output linguistic variable; $Y^{n}$ is chosen from $Y_{F M}, Y_{A N N}, Y_{L R}$, etc., which are the predicted results using various sub-models, such as the Fuzzy Models (FM), the Artificial Neural Network (ANN) [8] models, the Linear Regression (LR) [9] models, etc.

Fig. 2 shows an example of a two-dimensional modelling problem. In this example, the modelling space is divided into several parts. For every part, the most appropriate systems model(s) is (are) assigned. If a master fuzzy rule is used to describe the situation of the upper right sub-space in Fig. 2, then the master fuzzy rule will be as follows: 
IF $x_{1}$ is big and $x_{2}$ is big, THEN $y$ is $Y_{A N N}$,

where $Y_{A N N}$ is the predicted result using the neural-network sub-model.

$<$ Figure 2 $>$

\subsection{An approach of generating master fuzzy systems}

The development of the proposed master fuzzy system can be broadly divided into the following two stages:

1. Constructing several separate data-driven models using different modelling strategies, such as fuzzy modelling, artificial neural network, and linear regression. All these models work as sub-models of the whole modelling framework and they share the same training, validation, and testing data sets.

2. Constructing the 'master fuzzy system'.

For the second stage, one modified hierarchical clustering algorithm [4] is employed, which was designed to reduce the computation complexity and improve the efficiency. The algorithm has been shown to perform better than other well-known clustering algorithms [10], such as the fuzzy c-means (FCM) clustering algorithm [11], in the fuzzy model generation. The obtained cluster information is first used to define the fuzzy sets of the master fuzzy system and then used to elicit the related master fuzzy rules. The details of the whole modelling process can be described as follows:

\subsubsection{Obtaining cluster information:}

One should divide the input data of the training set into a set of clusters (sub-space) $\boldsymbol{C}_{n}\left(n=1,2, \ldots, N_{c}, N_{c}\right.$ is the number of clusters) using a clustering algorithm [10]. For every cluster (sub-space), the input data included in it are $\left\{\boldsymbol{p}^{n 1}, \boldsymbol{p}^{n 2}, \ldots, \boldsymbol{p}^{n(N D n)}\right\}$, where $\boldsymbol{p}^{n j}=\left[x_{1}{ }^{n j} x_{2}{ }^{n j} \ldots x_{D}{ }^{n j}\right]^{\mathrm{T}}, j=1,2, \ldots, N D n, N D n$ is the number of data in the $n$th cluster. For these input data, their corresponding output data are $\left\{y^{n 1}, y^{n 2}, \ldots, y^{n(N D n)}\right\}$.

\subsubsection{Defining fuzzy sets:}

For every master fuzzy rule, the parameters of the fuzzy sets $A_{i}^{n}\left(n=1,2, \ldots, N_{c} ; i=1\right.$, $2, \ldots, D ; D$ is the number of inputs) are obtained using the following method:

If Gaussian functions are used as the membership functions [3], i.e.

$$
\mu_{A_{i}^{n}}(x)=\exp \left(-\frac{\left(x-c_{i}^{n}\right)^{2}}{\sigma_{i}^{n^{2}}}\right),
$$


then $c_{i}^{n}$ can be calculated using the equation $[10,12]$

$$
c_{i}^{n}=\sum_{j=1}^{N D n} x_{i}^{n j} / N D n
$$

The idea behind this is that the centres of clusters are the centres of membership functions.

$\sigma_{i}^{n}$ can be calculated using the following equation $[10,12]$ :

$$
\sigma_{i}^{n}=\frac{\max _{j}\left(x_{i}^{n j}-c_{i}^{n}\right)}{\sqrt{-\ln (T h)}},
$$

where $j=1,2, \ldots, N D n, T h$ is a threshold value. This equation emanates from the principle that the membership function should cover all the data contained in its corresponding cluster. In other words, for every data included in one cluster, its membership degree should be high enough to ensure the data maps into this rule. Based on this requirement, the membership parameter $\sigma_{i}^{n}$ is designed to satisfy the following equation $[10,12]$ :

$$
\min _{j}\left(\mu_{A_{i}^{n}}\left(x_{i}^{n j}\right)\right)=\min _{j}\left(\exp \left(-\frac{\left(x_{i}^{n j}-c_{i}^{n}\right)^{2}}{\left(\sigma_{i}^{n}\right)^{2}}\right)\right)=T h
$$

where $j=1,2, \ldots, N D n$. This equation means that, for all the data included in the $n$th cluster, the membership degrees are higher than a threshold $T h$. The value of $T h$ can be set to 0.5 without any loss of generality. Equation (4) can be rewritten in the form of Equation (3).

\subsubsection{Generating the fuzzy rules:}

One master fuzzy rule corresponds to one data cluster. For a master fuzzy rule, its antecedent part is determined by the related cluster information [10] and the consequent part is generated through an error-oriented method. The principle behind lies in that the consequent of a master fuzzy rule is designed to be the prediction of the sub-model, which is most accurate in the situation that the premises represent. In particular, the consequent part of each rule is determined using the following methodology:

For every cluster (sub-space), the sum of the absolute prediction errors of each modelling method is first calculated. For instance, for the $n$th cluster, the absolute error sum of the fuzzy sub-model can be described as follows:

$$
s_{F M}^{n}=\sum_{j=1}^{N D n}\left|y^{n j}-y_{F M}^{n j}\right|
$$

where $y_{F M}^{n j}$ is the predicted output using the fuzzy sub-model corresponding to the input data $\boldsymbol{p}^{n j}$.

The output of the sub-model, which has the minimum value of the absolute error sum, 
is then set as the output of the relevant fuzzy rule. For instance, for the $n$th fuzzy rule:

$$
R_{n} \text { : IF } x_{1} \text { is } A_{1}{ }^{n} \text { AND ... AND } x_{D} \text { is } A_{D}{ }^{n} \text {, THEN } y \text { is } Y^{n} \text {. }
$$

$Y^{n}$ is calculated using the following method

$$
Y^{n}=\left\{\begin{array}{c}
Y_{F M}, \text { if } \min \left(s_{F M}^{n}, s_{A N N}^{n}, s_{L R}^{n}\right)=s_{F M}^{n} \\
Y_{A N N}, \text { if } \min \left(s_{F M}^{n}, s_{A N N}^{n}, s_{L R}^{n}\right)=s_{A N N}^{n} \\
Y_{L R}, \text { if } \min \left(s_{F M}^{n}, s_{A N N}^{n}, s_{L R}^{n}\right)=s_{L R}^{n},
\end{array},\right.
$$

where $s_{F M}^{n}, s_{A N N}^{n}$ and $s_{L R}^{n}$ are the sum of the absolute errors of the fuzzy sub-model, the neural-network sub-model, and the linear-regression sub-model based on the data of the $n$th cluster, respectively.

\subsubsection{Improving accuracy:}

Based on a fixed rule-base, the master fuzzy system is improved in terms of accuracy by optimising the parameters of the membership functions (peaks and widths). In this paper, the related work is carried out by using a salient nature-inspired optimisation algorithm, Reduced Space Searching Algorithm (RSSA) [13, 14]. This algorithm originated from an idea which relates to a simple experience when humans search for an optimal solution to a 'real-life' problem, i.e. when humans search for a candidate solution given a certain objective, a large area tends to be scanned first; should one succeed in finding clues in relation to the predefined objective, then the search space is greatly reduced for a more detailed search. This algorithm has been validated using a set of benchmark problems and shown to perform better than some well-known heuristic optimisation algorithms, such as the Covariance Matrix Adaptation Evolution Strategy (CMA-ES) [15], the Generalised Generation Gap model with the Parent-Centric Recombination operator (G3+PCX) [16], and the new structure Particle Swarm Optimization (nPSO) with a dynamically varying population $[17,18]$.

\section{Experimental Studies}

In order to validate the effectiveness of the proposed modelling paradigm, the associated strategy was applied to the modelling of two benchmark problems, one is a problem of static non-linear system approximation and the other is a dynamic system identification problem.

In the following experiments, the sub-models consist of one fuzzy system, one artificial neural network [8], and one linear-regression model [9]. Fuzzy systems and artificial neural networks have been widely accepted to be robust techniques for datadriven modelling. In this paper, some modelling problems studied are clearly not linear as a whole, while the linear-regression model was still employed, because these problems may include partially linear area, where the linear-regression model becomes naturally more suitable. Other types of model architecture can indeed be 
included if need be within this particular scheme. The fuzzy sub-model is a TakagiSugeno-Kang (TSK) fuzzy system [19], which is generated using a subtractive clustering method [20] and trained using a hybrid learning algorithm introduced in [21]. The neural-network sub-model is a feed-forward back-propagation network [8]. For the optimisation algorithm RSSA, the configuration of parameters is inspired from suggestions included in [14]: $C_{1}=D / 2+8, C_{2}=1, K=0.5, m=20$, where $D$ is the dimension of the optimisation problem; the variation operator works as a combination of the one-dimensional variation strategy (with the $50 \%$ probability of usage) and the multi-dimensional variation strategy (with the $50 \%$ probability of usage). The Root Mean Square Error (RMSE) index works as the performance index of modelling accuracy.

\subsection{The non-linear function approximation}

In this experiment, the modelling target is to approximate the following two-input single-output non-linear system $[22,23]$ :

$$
y=\left(1+x_{1}^{-2}+x_{2}^{-1.5}\right)^{2}, 1 \leq x_{1}, x_{2} \leq 5 .
$$

In this case, 50 data points were used for training and another 50 randomly generated data points were used for model testing. The master fuzzy system consisted of 20 fuzzy rules. The fuzzy sub-model includes 6 fuzzy rules and the neural-network submodel includes a hidden layer of 2 neurons. The training epochs for both the fuzzy and neural-network sub-models were set to be 200. The maximum number of function evaluations for RSSA was set to 5,000.

The experiment was carried out using 20 runs. Table 1 shows the performance index values of the sub-models and the final integrated model. It can be seen that, for this problem, the proposed paradigm can successfully merge other modelling techniques. The result also reveals that the integrated model outperforms any of the sub-models in the prediction accuracy, because the integrated model has merged all the 'elite' parts of the sub-models.

$<$ Table 1>

As an example, one set of typical results out of the 20 runs is selected and shown in the following figures. Figs. 3 and 4 show the predicted outputs versus the measured outputs of the obtained model based on training data and testing data, respectively. They also include the performance of the sub-models. From these figures, one can observe that, in a certain local area, the integrated model performs similar with one of the sub-models, which always performs the best in all sub-models in this local area. This property is highly consistent with the initial design aim.

$<$ Figure $3>$ 
$<$ Figure 4>

For more details about the master fuzzy system, Fig. 5 also shows 5 rules out of the rule-base. It can be observed that the neural-network sub-model is fired in most of the rules, since it performs best in this particular case.

$<$ Figure 5 $>$

\subsection{The identification of a dynamic system}

In this problem, the modelling target is a non-linear second-order plant, which has been studied in $[24,25]$,

$$
y(k)=g(y(k-1), y(k-2))+u(k),
$$

where

$$
g(y(k-1), y(k-2))=\frac{y(k-1) y(k-2)(y(k-1)-0.5)}{1+y^{2}(k-1)+y^{2}(k-2)} .
$$

where $y()$ is the output of the system; $g()$ is a non-linear component; $u()$ is the input signal; $k$ is the index of the input signals. The output of this system depends on both its past states and the current input. The modelling purpose is to approximate the nonlinear component $g(y(k-1), y(k-2))$.

Following the experimental settings in [25], 400 simulated data samples were generated from the plant model (8). With the starting equilibrium state $(0,0)$, the first 200 samples of training data were obtained by using a random input signal $u(k)$ that is uniformly distributed in the interval $[-1.5,1.5]$ and the rest 200 samples of testing data were obtained by using a sinusoidal input signal $u(k)=\sin (2 \pi k / 25)$. In this case, the master fuzzy system consists of 20 fuzzy rules. The fuzzy sub-model includes 4 fuzzy rules and the neural-network sub-model includes a hidden layer of 3 neurons. The training epochs for both the fuzzy and neural-network sub-models were set to be 200 . The maximum number of function evaluations for RSSA was set to 5,000.

This experiment was repeated 20 times. Table 2 shows the performance index values, RMSE(s) of the sub-models and the final integrated model. It can be seen that the proposed modelling approach can successfully combine different modelling techniques and the integrated model outperforms any of the sub-models in accuracy.

$<$ Table 2>

One typical model out of the 20 runs is selected as an example. Figs. 6 and 7 show the prediction performance of this model based on the training data and the testing data, respectively. To provide more details relating to the master fuzzy system, 5 master fuzzy rules are shown in Fig. 8. 
$<$ Figure 6>

$<$ Figure $7>$

$<$ Figure $8>$

\section{Applications to Engineering Materials}

In material engineering and mechanical engineering, it is essential to establish accurate and reliable prediction models for various material behaviours [26, 27]. But it may be 'tricky' to precisely describe these behaviours using mathematical models alone due to the complexity of materials' chemical composites and their underlying physical processing mechanisms, such as heat treatment and machining.

In this work, the proposed modelling approach was applied to two such industrial problems, the prediction of machining induced residual stresses in aerospace alloy components and the prediction of mechanical properties of alloy steels, including Ultimate Tensile Strength (UTS) and elongation. In the following experiments, the configuration of all the parameters was set to be the same as that used in Section 3, where the sub-models consist of one TSK fuzzy system [19, 20, 21], one feed-forward neural network [8], and one linear-regression model [9].

\subsection{Prediction of machining induced residual stresses}

The residual stresses induced during shaping and machining play an important role in determining the integrity and durability of metal components [28]. Their combination with primary loads contributes to changes in the operating performance of mechanical parts. Tensile residual stresses enhance the likelihood of fatigue, fracture and corrosion induced failures. Conversely, compressive residual stresses are often introduced by shot-peening and burnishing to enhance structural integrity and durability [29]. An important issue of producing safety critical components is to find the machining parameters that create compressive surface stresses or minimise tensile surface stresses.

Metal removal by machining operations such as milling and drilling induces residual stresses in the near surface region. These stresses are highly dependent on the machining parameters and cannot be accurately described using mathematical models because of the high complexity of the processes. Finite Element Methods (FEMs) have been widely used to investigate this phenomenon [30]. The drawbacks of FEM approaches relate to the long time needed for the solution of complex models and their inability to learn from examples.

In recent years, some intelligent data-driven modelling approaches have been considered for the prediction of residual stresses. For instance, artificial neural 
networks have been used by Kafkas et al. [31] and Umbrello et al. [32], and fuzzy rule-based systems have been employed by Zhang et al. [33, 34]. Compared with analytically based methods, such as FEMs, intelligent systems are simpler in structure and easier to apply. They are capable of learning from data without needing much prior knowledge about the materials and machining processes. They are also convenient when combined with optimisation techniques to identify the input parameters that will provide a desirable residual stress profile.

Our research programme proposes to investigate manufacturing induced part distortion in aerospace alloy components, where part distortion is a function of residual stress and is caused by the complex combination of material processing, or the complex interaction between material processing. In aerospace industry, excessive distortion may result in the rejection of a part as well as costly and time-consuming rework before placement in service. It is reported that tens of millions of Euros are spent every year in an attempt to either avoid or remedy distortion in components [35]. In this project, the prediction of machining induced residual stresses can be conducted using the systems modelling approach introduced in this paper. Fig. 9 shows a predicted part distortion under residual stresses using finite element modelling combined with the developed prediction models [34].

$<$ Figure 9 $>$

In the following case, the proposed approach was applied to predict the surface and near-surface residual stresses (up to $250 \mu \mathrm{m}$ in depth) in aerospace aluminium parts, where experimental tests were conducted by the Institute of Production Engineering and Machine Tools (IFW), the University of Hannover using the X-ray diffraction measurements.

In this case, 194 residual stress data were used for training and 49 data were used for final testing. System inputs include the profiles of machining parameters, i.e. cutting speed, feed per tooth, feed velocity, as well as coolant medium and measurement depth. The residual stress in the longitudinal rolling direction of the original aluminium billet is the modelling target.

In the following experiments, the fuzzy sub-model includes 20 fuzzy rules, the neuralnetwork sub-model includes a hidden layer of 5 neurons, and the master fuzzy system includes 50 rules. The training epochs for both the fuzzy and neural-network submodels were set to be 200 . The maximum number of function evaluations for RSSA was set to 5,000 .

The experiment was carried out over 20 runs. Table 3 includes the main parameters of the final integrated model as well as three sub-models. One set of results out of the 20 runs is selected and shown as follows. Fig. 10 shows the predicted outputs versus the measured outputs of the obtained model and different sub-models based on the testing data. It can be observed that the proposed modelling approach can successfully combine other modelling techniques and the integrated model outperforms any of the 
sub-models.

$<$ Table 3>

$<$ Figure 10>

By exploiting the generated model, residual stress curves can also be obtained. This is achieved by plotting one input variable, i.e. measurement depth, against the output, residual stress, while keeping other input variables constant. Fig. 11 shows both the predicted curves and the measured data. It allows us to observe the fact that the integrated model predicts the residual stress more accurately than the sub-models.

$<$ Figure $11>$

To provide more information about the above models, Fig. 12 shows 3 master fuzzy rules out of the rule-base. It should also be noted that, in this experiment, the submodels were not well optimised, while the integrated model shows a clear improvement in accuracy performance. This means that the proposed method can save a lot of time and effort normally used in models' training and optimisation.

$<$ Figure 12>

\subsection{Prediction of mechanical properties}

In material engineering, specialist heat treatments consist of two main stages: hardening and tempering, are used to develop the required mechanical properties in a range of alloy steels [36]. It is not possible to accurately describe the process behaviour using mathematical models alone due to the complexity of the underlying physical mechanisms. In this work, two typical mechanical properties of heat-treated alloy steels are studied, i.e. UTS and elongation [37], both of which are obtained via an engineering tension test. The UTS represents a measure of the maximum load that a material can withstand. The elongation is a measure of ductility, which is usually expressed as a percentage change in the gauge length or diameter of the specimen after fracture [37]. In the following experiments, all the data had previously been provided by Tata Steel Europe.

\subsubsection{Ultimate tensile strength}

In the first case, 3760 UTS data were used for data-driven modelling. $60 \%$ of the data were used for training, $20 \%$ of the data were used for validation, and the remaining $20 \%$ were used for final testing. These UTS data include 15 inputs and one output, which is considered to be a high-dimensional problem for modelling purposes. The inputs consist of the weight percentages for the chemical composites, namely Carbon (C), Silica (Si), Manganese (Mn), Sulphur (S), Chromium (Cr), Molybdenum (Mo), 
Nickel (Ni), Aluminium (Al), and Vanadium (V), the test depth, the size and site where the processing of the alloy steel took place, the cooling medium, as well as the hardening and tempering temperatures.

In this experiment, the fuzzy sub-model includes 8 fuzzy rules, the neural-network sub-model includes a hidden layer of 5 neurons, and the master fuzzy system includes 50 fuzzy rules. The training epochs for both the fuzzy and neural-network sub-models were set to be 200. The maximum number of function evaluation for RSSA was set to 1,000 .

The experiment was repeated 20 times. Table 4 shows the performance index values of the final integrated model as well as the sub-models. One of the 20 models is selected and shown in the following figures. Fig. 13 shows the prediction performance of the elicited models based on the testing data.

$<$ Table 4>

$<$ Figure 13>

Fig. 14 shows the three-dimensional response surfaces of the obtained UTS model. These surfaces are achieved by plotting two varying input variables against the output while keeping other input variables constant. The constant variables are set to the average values of the dominant steel grade, which is the $1 \% \mathrm{CrMo}$ steel grade [36]. It can be seen that the integrated model shows a combination of the sub-models' characters. This combination may correct any errors of mapping generated by the submodels.

$<$ Figure 14>

\subsubsection{Elongation}

In the second case, 3710 Elongation data were used, $60 \%$ of the data were used for training, $20 \%$ of them were used for validation, and the remaining $20 \%$ were used for final testing. The inputs consist of the weight percentages for Carbon (C), Silica (Si), Manganese (Mn), Sulphur (S), Chromium (Cr), Molybdenum (Mo), Nickel (Ni), Aluminium (Al), and Vanadium (V), the test depth, the size and site where the processing of the alloy steel took place, the cooling medium, the elongation gauge length, as well as the hardening, tempering, and impact test temperatures.

The fuzzy sub-model includes 9 fuzzy rules, the neural-network sub-model includes a hidden layer of 20 neurons, and the master fuzzy system includes 50 rules. The training epochs for both the fuzzy and neural-network sub-models were set to be 200 . The maximum number of function evaluation for RSSA was set to 1,000.

The experiment was run 20 times. Table 5 describes the RMSE values of the submodels and the final integrated model. It can be seen that, for this industrial modelling 
problem, the proposed modelling approach can successfully merge other modelling techniques and the integrated model outperforms any of the sub-models in prediction accuracy.

$<$ Table 5>

One model is selected and shown in the following figures. Fig. 15 shows the prediction performance of the obtained models on the testing data. It can be seen that, in a certain local area, the integrated model performs similar with one of the submodels, which always performs the best in all sub-models in this local area.

$<$ Figure 15 $>$

Fig. 16 shows the three-dimensional response surfaces of the elicited elongation model, where the constant variables are set to the $1 \%$ CrMo steel grade [36]. It can be observed that the integrated model shows a combination of the sub-models' characters, which may correct any errors of mapping generated by the sub-models. It should also be noted that, in this experiment, the sub-models were also not well optimised, but the integrated model shows a clear improvement in accuracy performance. It means that the proposed method can save a lot of time and effort used in models' training and optimisation.

$<$ Figure 16>

\section{Conclusions}

In this paper, a new structure-free modelling paradigm was proposed by systematically combining various modelling techniques. This new approach does not rely on a fixed modelling structure and can include the advantages of different modelling techniques. It does not need the involved sub-models to be optimised, which can save a lot of time and effort used in training and optimisation. The proposed approach has been validated using benchmark problems, and successfully applied within the context of eliciting the prediction models for machining induced residual stresses and mechanical properties of alloys. In future, the proposed paradigm can be further enhanced via the introduction of a heuristic learning mechanism when generating master fuzzy rules. By doing so, not only accuracy but reliability can also be considered in the control of sub-models' fusion.

\section{Acknowledgements}

The authors wish to thank the anonymous reviewers for their comments which helped to improve the quality of this paper. They wish to acknowledge Drs Luis de Leon, Soufiene Boumaiza and Richard J. Greene for their help in coordinating the data collection process. They also wish to acknowledge the financial support for this work 
from the European Union under the Framework 6 initiative and the UK-EPSRC under Grant Reference Number: EP/E063497/1.

\section{References:}

[1] L.A. Zadeh, Fuzzy sets, Information and control 8 (3) (1965), pp. 338-353.

[2] L.A. Zadeh, Outline of a new approach to the analysis of complex systems and decision processes, IEEE Transactions on systems, man, and cybernetics 3 (1973), pp. 28-44.

[3] L.-X. Wang, A course in fuzzy systems and control. Prentice-Hall, Englewood Cliffs, NJ, USA, 1997.

[4] Q. Zhang, M. Mahfouf, Mamdani-type fuzzy modelling via hierarchical clustering and multi-objective particle swarm optimisation (FM-HCPSO), International journal of computational intelligence research, 4 (4) (2008), pp. 314-328.

[5] Q. Zhang, M. Mahfouf, A hierarchical mamdani-type fuzzy modelling approach with new training data selection and multi-objective optimisation mechanisms: a special application for the prediction of mechanical properties of alloy steels, Applied soft computing, 11 (2) (2011), pp. 2419-2443.

[6] E.H. Mamdani, S. Assilian, An experiment in linguistic synthesis with a fuzzy logic controller, International Journal of Man-Machine Studies 7 (1) (1975), pp. $1-13$.

[7] M. Sugeno, On stability of fuzzy systems expressed by fuzzy rules with singleton consequents, IEEE Transactions on Fuzzy Systems 7 (2) (1999), pp. 201-224.

[8] S. Haykin, Neural networks: a comprehensive foundation, Prentice Hall, 1999.

[9] D.C. Montgomery, E.A. Peck, G.G. Vining, Introduction to linear regression analysis, 4th edition, Wiley, 2006.

[10] Q. Zhang, Nature-Inspired Multi-Objective Optimisation and Transparent Knowledge Discovery via Hierarchical Fuzzy Modelling. PhD Thesis, The Department of Automatic Control and Systems Engineering, The University of Sheffield, UK, 2008.

[11] J.C. Bezdek, Pattern Recognition with Fuzzy Objective Function Algoritms, Plenum Press, New York, 1981.

[12] Q. Zhang, M. Mahfouf, Fuzzy predictive modelling using hierarchical clustering and multi-objective optimisation for mechanical properties of alloy steels, Proceedings of the 12th IFAC Symposium on Automation in Mining, Mineral and Metal Processing, 1 (1), pp. 427-432, 2007, doi: 10.3182/20070821-3-CA2919.00059.

[13] Q. Zhang, M. Mahfouf, A new reduced space searching algorithm (RSSA) and its application in optimal design of alloy steels, Proceedings of the 2007 IEEE Congress on Evolutionary Computation, pp. 1815-1822, 2007.

[14] Q. Zhang, M. Mahfouf, A nature-inspired multi-objective optimisation strategy based on a new reduced space searching algorithm for the design of alloy steels, Engineering applications of artificial intelligence, 23 (5) (2010), pp. 660-675. 
[15] N. Hansen, S.D. Muller, P. Koumoutsakos, Reducing the time complexity of the derandomized evolution strategy with covariance matrix adaptation (CMA-ES), Evolutionary Computation 11 (1) (2003), pp. 1-18.

[16] K. Deb, A. Anand, D. Joshi, A computationally efficient evolutionary algorithm for real-parameter optimisation, Evolutionary Computation 10 (4), (2002), pp. 371-395.

[17] Q. Zhang, M. Mahfouf, A new structure for particle swarm optimization (nPSO) applicable to single objective and multiobjective problems, Proceedings of the 3rd International IEEE Conference on Intelligent Systems, pp. 176-181, 2006.

[18] Q. Zhang, M. Mahfouf, A modified PSO with a dynamically varying population and its application to the multi-objective optimal design of alloy steels, Proceedings of the 2009 IEEE Congress on Evolutionary Computation, pp. 3241-3248, 2009.

[19] T. Takagi, M. Sugeno, Fuzzy identification of systems and its applications to modeling and control, IEEE Transaction Systems, Man and Cybernetics 15 (1) (1985), pp. 116-132.

[20] S. Chiu, Fuzzy model identification based on cluster estimation, Journal of intelligent and fuzzy systems, 2 (3) (1994), pp. 267-278.

[21] J.-S.R. Jang, ANFIS: adaptive-network-based fuzzy inference systems, IEEE transactions on systems, man, and cybernetics, 23 (3) (1993), pp. 665-685.

[22] M. Sugeno, T. Yasukawa, A fuzzy-logic-based approach to qualitative modeling, IEEE Transactions on Fuzzy Systems 1 (1) (1993), pp. 7-31.

[23] I. Rojas, H. Pomares, J. Ortega, A. Prieto, Self-organized fuzzy system generation from training examples, IEEE Transactions on Fuzzy Systems 8 (1) (2000), pp. 23-36.

[24] J. Yen, L. Wang, Simplifying fuzzy rule-based models using orthogonal transformation methods, IEEE Transactions on Systems, Man, and Cybernetics - Part B: Cybernetics 29 (1) (1999), pp. 13-24.

[25] M. Setnes, H. Roubos, GA-fuzzy modeling and classification: complexity and performance, IEEE Transactions on Fuzzy Systems 8 (5) (2000), pp. 509-522.

[26] Q. Zhang, M. Mahfouf, G. Panoutsos, K. Beamish, I. Norris, Multiple characterisation modelling of friction stir welding using a multi-objective datadriven fuzzy modelling approach, Proceedings of the 2011 IEEE International Conference on Fuzzy Systems, pp. 2288-2295, 2011.

[27] Q. Zhang, M. Mahfouf, Fuzzy modelling using a new compact fuzzy system: a special application for the mechanical property prediction of alloy steel, Proceedings of the 2011 IEEE International Conference on Fuzzy Systems, pp. 1041-1048, 2011.

[28] British Energy Generation Ltd. Assessment of the integrity of structures containing defects, R6 revision 4, 2006.

[29] R.C. McClung, A literature survey on the stability and significance of residual stresses during fatigue, Fatigue and fracture of engineering materials and structures, 30 (2007), pp. 173-205.

[30] K.C. Ee, O.W. Dillon Jr., I.S. Jawahir, Finite Element Modeling of Residual 
Stresses in Machining Induced by Cutting using a Tool with Finite Edge Radius, International Journal of Mechanical Sciences, 47 (2005), pp. 1611-1628.

[31] F. Kafkas, C. Karatas, A. Sozen, E. Arcaklioglu, S. Saritas, Determination of Residual Stresses based on Heat Treatment Conditions and Densities on a Hybrid (FLN2-44-5) Powder Metallurgy Steel using Artificial Neural Network, Materials and Design, 28 (2007), pp. 2431-2442.

[32] D. Umbrello, G. Ambrogio, L. Filice, R. Shivpuri, A Hybrid Finite Element Method-Artificial Neural Network Approach for Predicting Residual Stresses and the Optimal Cutting Conditions during Hard Turning of AISI 52100 Bearing Steel. Materials and Design, 29 (2008), pp. 873-883.

[33] Q. Zhang, M. Mahfouf, L. de Leon, S. Boumaiza, J.R. Yates, C. Pinna, R.J. Greene, Prediction of machining induced residual stresses in aluminium alloys using a hierarchical data-driven fuzzy modelling approach, Proceedings of the IFAC Workshop on Automation in Mining, Mineral and Metal Industry (2009), 1 (1), 2009, doi: 10.3182/20091014-3-CL-4011.00042.

[34] Q. Zhang, M. Mahfouf, J.R. Yates, C. Pinna, G. Panoutsos, S. Boumaiza, R.J. Greene, L. de Leon, Modeling and optimal design of machining-induced residual stresses in aluminium alloys using a fast hierarchical multiobjective optimization algorithm, Materials and manufacturing processes, 26 (3) (2011), pp. 508-520.

[35] W.M. Sim, Challenges of Residual Stress and Part Distortion in the Civil Airframe Industry, In Proceedings of the 2nd International Conference on Distortion Engineering, 2008.

[36] J. Tenner, Optimisation of the Heat Treatment of Steel Using Neural Networks, $\mathrm{PhD}$ Thesis, The Department of Automatic Control and Systems Engineering, The University of Sheffield, UK, 1999.

[37] G.E. Dieter, Mechanical metallurgy: SI metric edition, McGraw-Hill, London, UK, 1988. 


\section{Tables:}

Table 1. Training and testing errors for the non-linear function approximation problem

\begin{tabular}{|c|c|c|}
\hline & $\begin{array}{c}\text { Root mean square error of } \\
\text { training } \\
\text { (mean } \pm \text { standard } \\
\text { deviation) }\end{array}$ & $\begin{array}{c}\text { Root mean square error of } \\
\text { testing } \\
\text { (mean } \pm \text { standard } \\
\text { deviation) }\end{array}$ \\
\hline Fuzzy Sub-model & $0.0554 \pm 0.0055$ & $0.1373 \pm 0.0226$ \\
\hline $\begin{array}{c}\text { Neural-network Sub- } \\
\text { model }\end{array}$ & $0.1248 \pm 0.1386$ & $0.1479 \pm 0.1487$ \\
\hline $\begin{array}{c}\text { Linear-regression Sub- } \\
\text { model }\end{array}$ & $0.5521 \pm 0.0155$ & $0.5691 \pm 0.1613$ \\
\hline Integrated model & $0.0473 \pm 0.0043$ & $0.0805 \pm 0.0315$ \\
\hline
\end{tabular}

Table 2. Training and testing errors for the dynamical system identification problem

\begin{tabular}{|c|c|c|}
\hline & $\begin{array}{c}\text { Root mean square error of } \\
\text { training } \\
\text { (mean } \pm \text { standard } \\
\text { deviation) }\end{array}$ & $\begin{array}{c}\text { Root mean square error of } \\
\text { testing } \\
\text { (mean } \pm \text { standard } \\
\text { deviation) }\end{array}$ \\
\hline Fuzzy Sub-model & $0.0552 \pm 0.0326$ & $0.0497 \pm 0.0249$ \\
\hline $\begin{array}{c}\text { Neural-network Sub- } \\
\text { model }\end{array}$ & $0.0576 \pm 0.0523$ & $0.0517 \pm 0.0351$ \\
\hline $\begin{array}{c}\text { Linear-regression Sub- } \\
\text { model }\end{array}$ & $0.2218 \pm 0.0092$ & $0.1708 \pm 0.0197$ \\
\hline Integrated model & $0.0280 \pm 0.0213$ & $0.0219 \pm 0.0092$ \\
\hline
\end{tabular}

Table 3. Training and testing errors for the prediction of the machining induced residual stress

\begin{tabular}{|c|c|c|}
\hline & $\begin{array}{c}\text { Root mean square error of } \\
\text { training } \\
\text { (mean } \pm \text { standard } \\
\text { deviation) }\end{array}$ & $\begin{array}{c}\text { Root mean square error of } \\
\text { testing } \\
\text { (mean } \pm \text { standard } \\
\text { deviation) }\end{array}$ \\
\hline Fuzzy Sub-model & $21.2384 \pm 3.2833$ & $31.5351 \pm 3.4091$ \\
\hline $\begin{array}{c}\text { Neural-network Sub- } \\
\text { model }\end{array}$ & $17.0670 \pm 1.7436$ & $27.1175 \pm 3.6044$ \\
\hline $\begin{array}{c}\text { Linear-regression Sub- } \\
\text { model }\end{array}$ & $84.6009 \pm 40.7627$ & $105.5339 \pm 13.4400$ \\
\hline Integrated model & $13.4949 \pm 0.9315$ & $22.8968 \pm 2.0986$ \\
\hline
\end{tabular}


Table 4. Training and testing errors for the UTS prediction

\begin{tabular}{|c|c|c|}
\hline & $\begin{array}{c}\text { Root mean square error of } \\
\text { training } \\
\text { (mean } \pm \text { standard } \\
\text { deviation) }\end{array}$ & $\begin{array}{c}\text { Root mean square error of } \\
\text { testing } \\
\text { (mean } \pm \text { standard } \\
\text { deviation) }\end{array}$ \\
\hline Fuzzy Sub-model & $35.5840 \pm 0$ & $42.4510 \pm 0$ \\
\hline $\begin{array}{c}\text { Neural-network Sub- } \\
\text { model }\end{array}$ & $37.0458 \pm 1.2144$ & $40.4127 \pm 0.8832$ \\
\hline $\begin{array}{c}\text { Linear-regression Sub- } \\
\text { model }\end{array}$ & $55.5079 \pm 0$ & $56.9017 \pm 0$ \\
\hline Integrated model & $33.7989 \pm 0.7481$ & $38.4084 \pm 0.6668$ \\
\hline
\end{tabular}

Table 5. Training and testing errors for the elongation prediction

\begin{tabular}{|c|c|c|}
\hline & $\begin{array}{c}\text { Root mean square error of } \\
\text { training } \\
\text { (mean } \pm \text { standard } \\
\text { deviation) }\end{array}$ & $\begin{array}{c}\text { Root mean square error of } \\
\text { testing } \\
\text { (mean } \pm \text { standard } \\
\text { deviation) }\end{array}$ \\
\hline Fuzzy Sub-model & $1.3431 \pm 0$ & $1.5317 \pm 0$ \\
\hline $\begin{array}{c}\text { Neural-network Sub- } \\
\text { model }\end{array}$ & $1.2944 \pm 0.0971$ & $1.4717 \pm 0.0954$ \\
\hline $\begin{array}{c}\text { Linear-regression Sub- } \\
\text { model }\end{array}$ & $2.6149 \pm 0$ & $2.2741 \pm 0$ \\
\hline Integrated model & $1.2707 \pm 0.0682$ & $1.4210 \pm 0.0332$ \\
\hline
\end{tabular}


Figures:

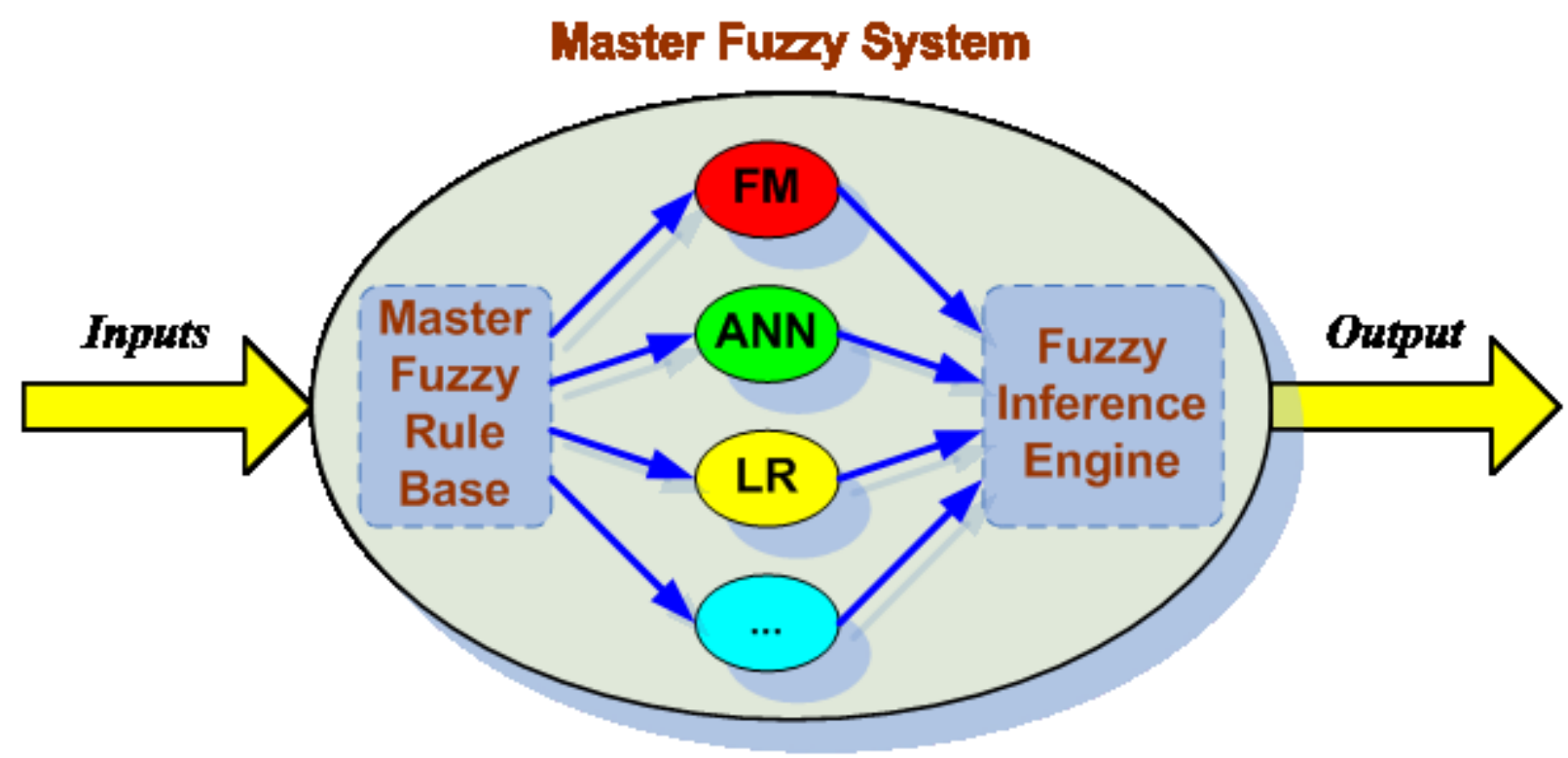

Fig. 1. The diagram of the master fuzzy system.

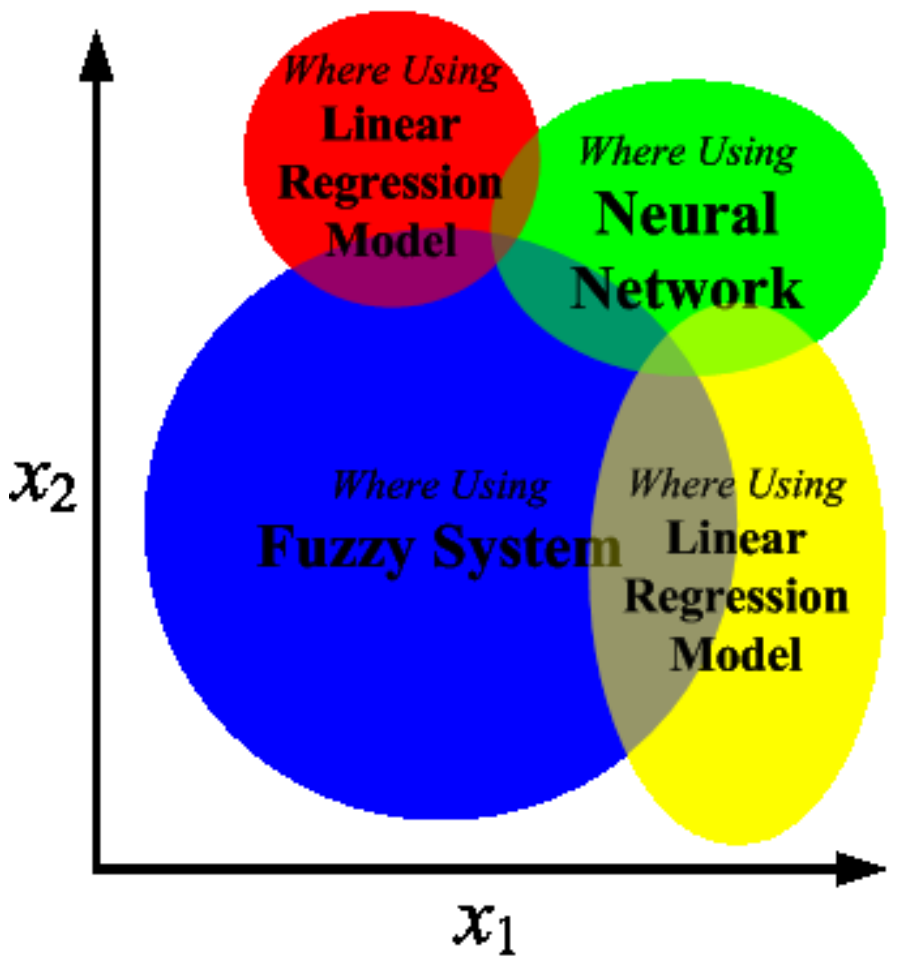

Fig. 2. An example of employing various methods in one modelling problem. 


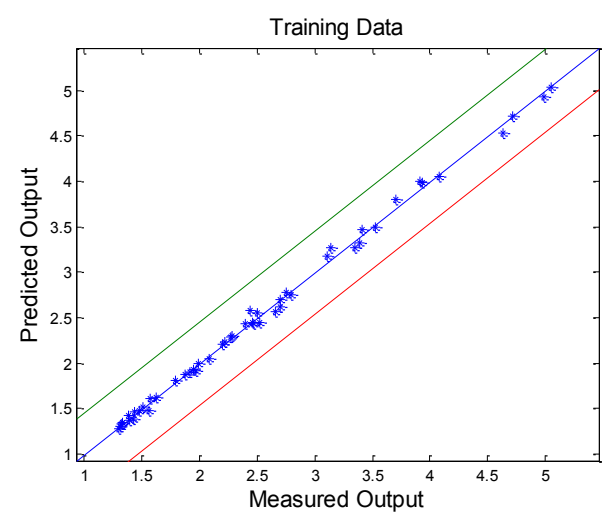

(a)

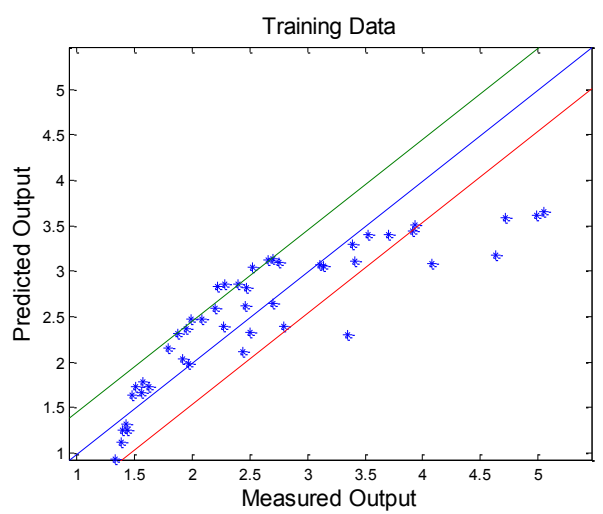

(c)

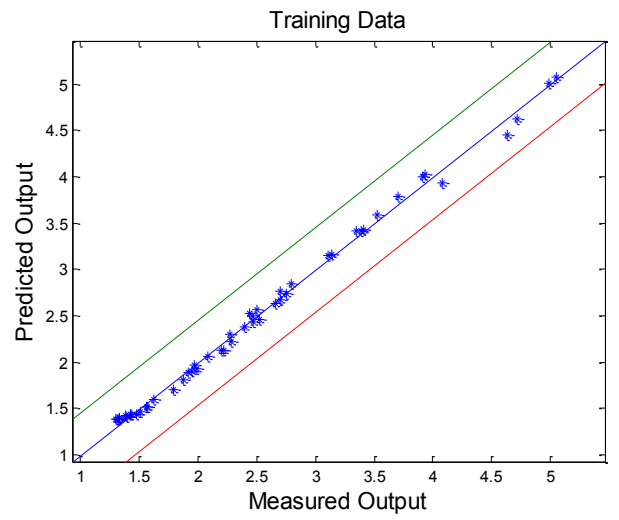

(b)

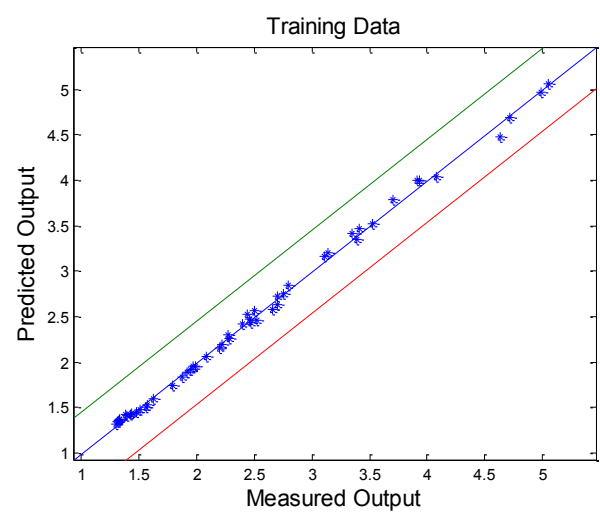

(d)

Fig. 3. The models' predicted outputs versus measured outputs based on the training data of the non-linear function approximation problem (with the $+10 \%$ and $-10 \%$ error bands): (a) the fuzzy sub-model, (b) the neural-network sub-model, (c) the linear-regression sub-model, and (d) the integrated model of the above sub-models. 


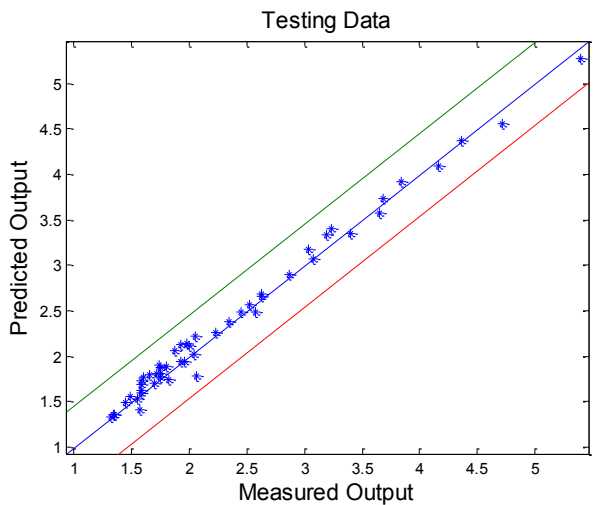

(a)

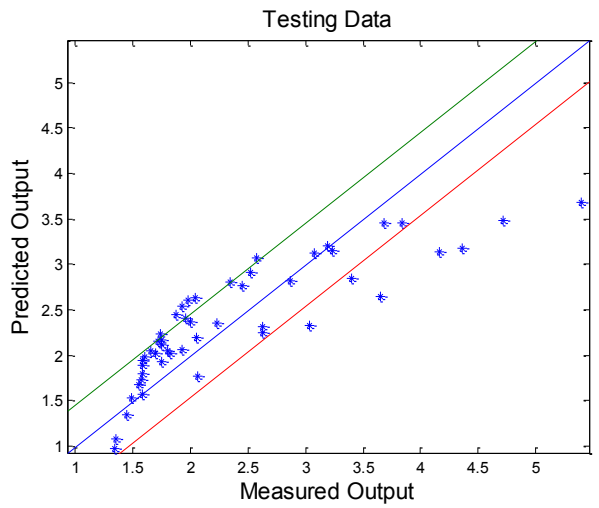

(c)

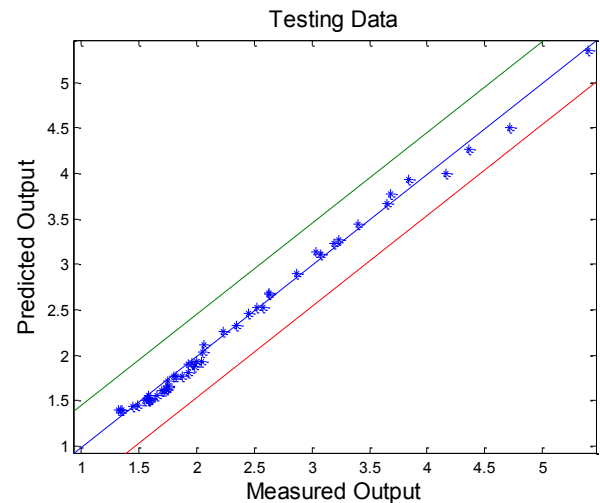

(b)

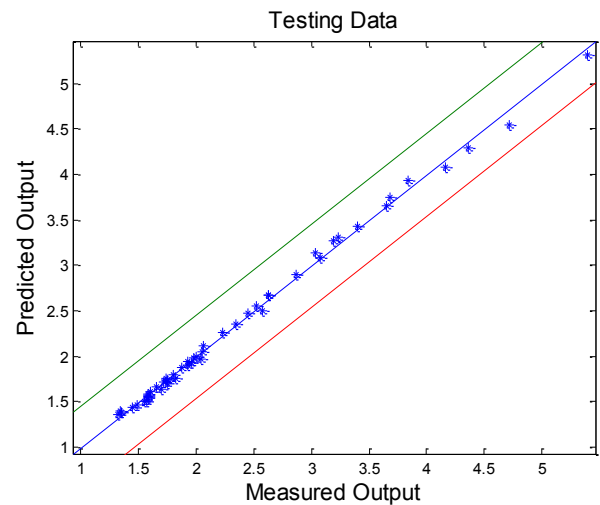

(d)

Fig. 4. The models' predicted outputs versus measured outputs based on the testing data of the non-linear function approximation problem (with the $+10 \%$ and $-10 \%$ error bands): (a) the fuzzy sub-model, (b) the neural-network sub-model, (c) the linear-regression sub-model, and (d) the integrated model. 


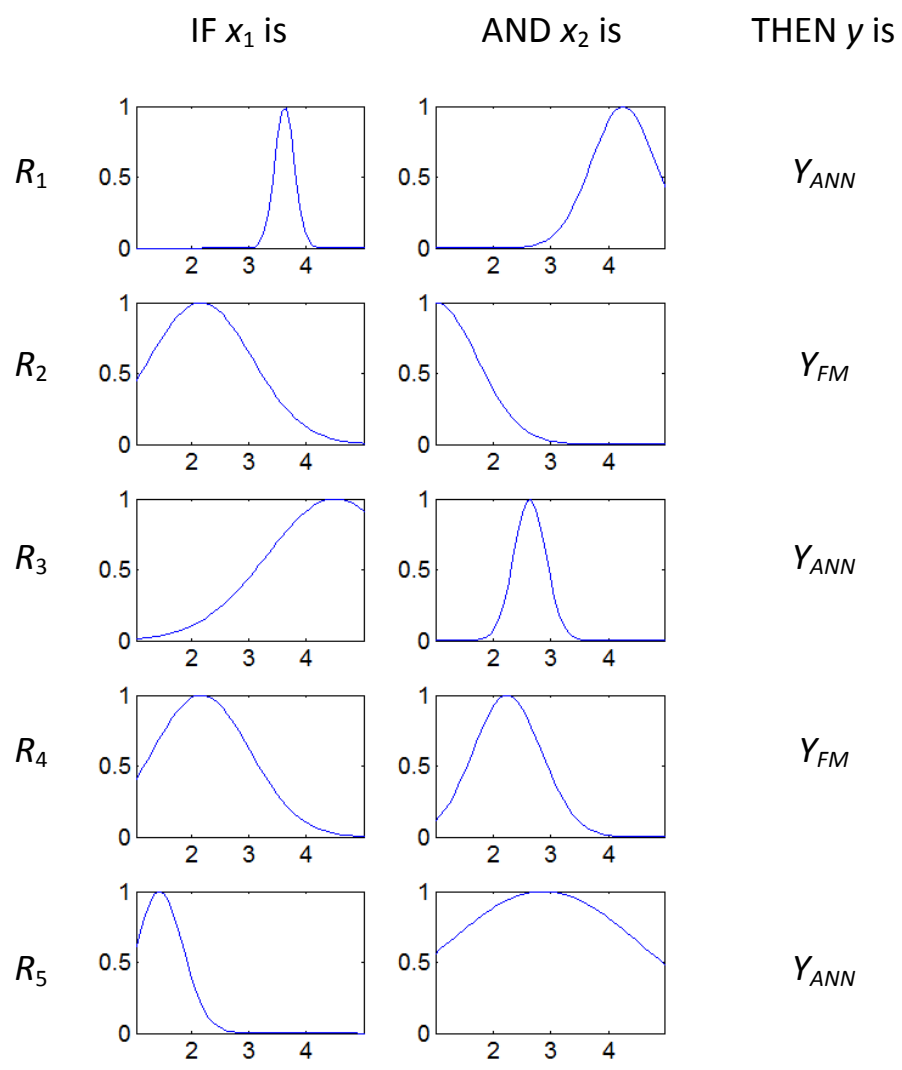

Fig. 5. Master fuzzy rules for the non-linear function approximation problem. 


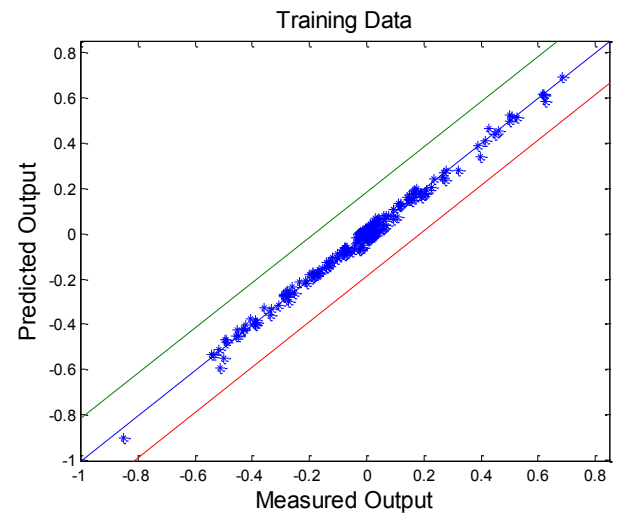

(a)

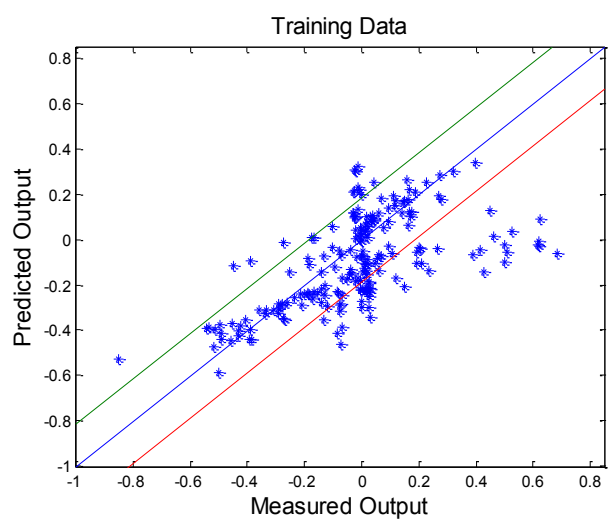

(c)

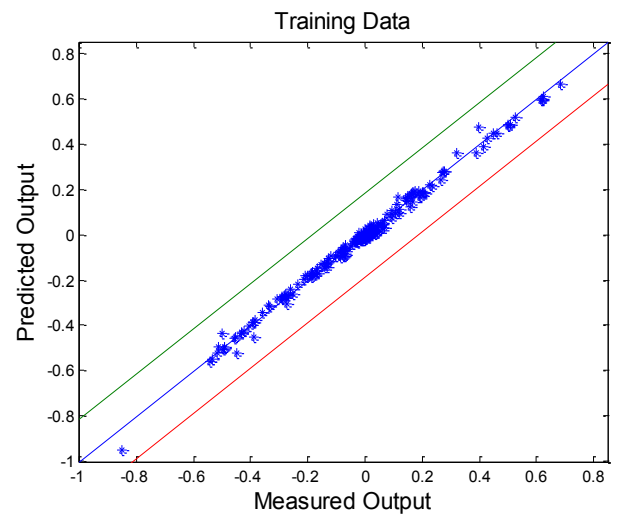

(b)

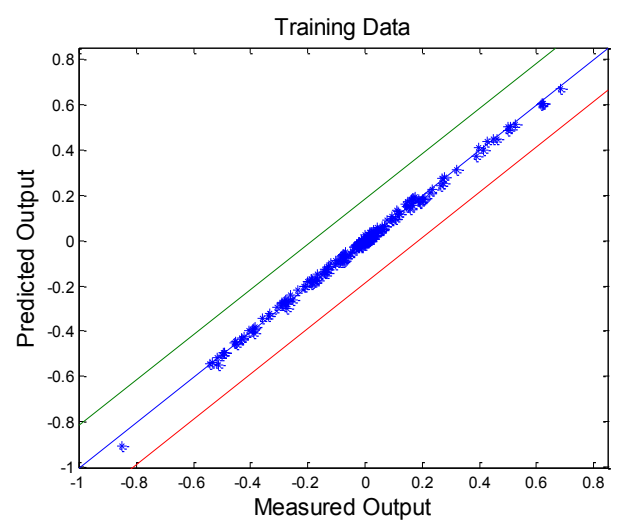

(d)

Fig. 6. The models' predicted outputs versus measured outputs based on the training data of the dynamical system identification problem (with the $+10 \%$ and $-10 \%$ error bands): (a) the fuzzy sub-model, (b) the neural-network sub-model, (c) the linear-regression sub-model, and (d) the integrated model. 


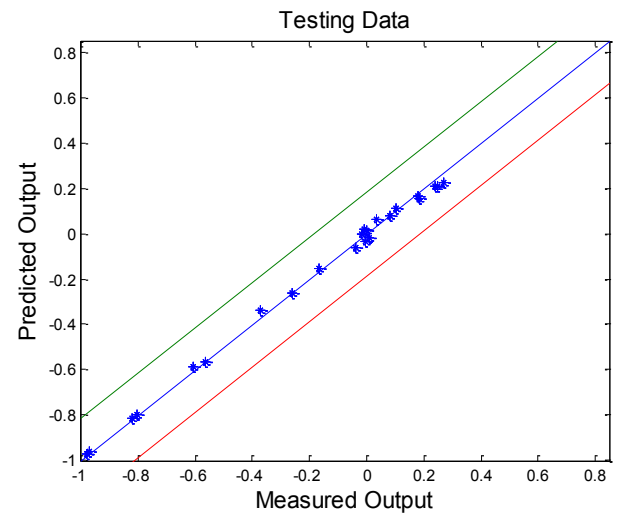

(a)

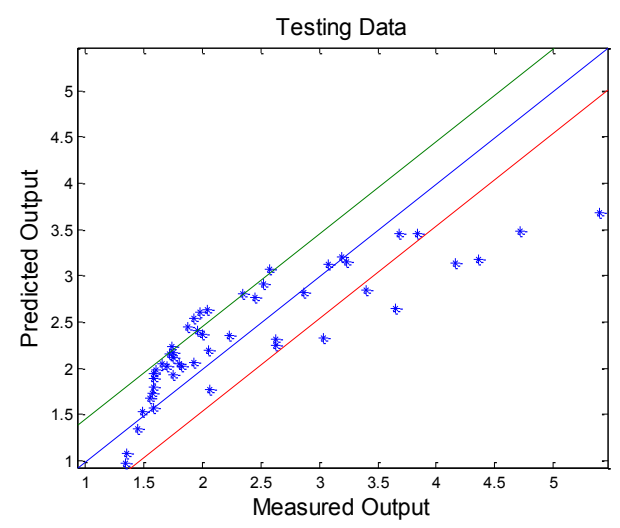

(c)

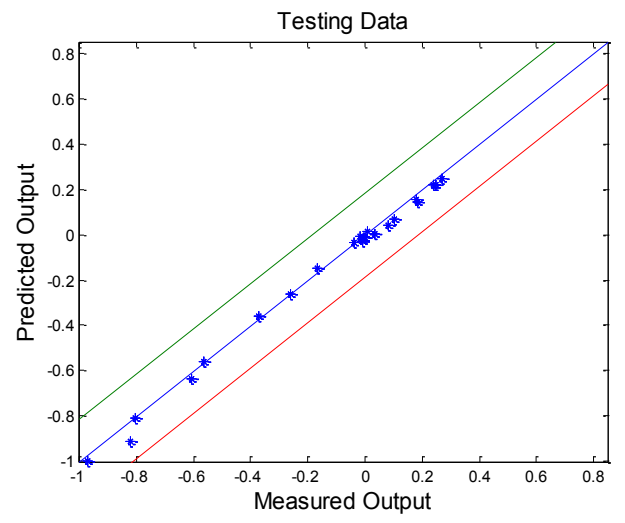

(b)

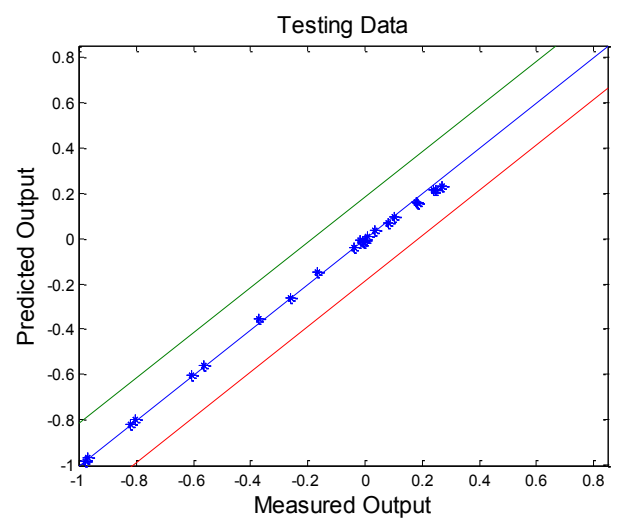

(d)

Fig. 7. The models' predicted outputs versus measured outputs based on the testing data of the dynamical system identification problem (with the $+10 \%$ and $-10 \%$ error bands): (a) the fuzzy sub-model, (b) the neural-network sub-model, (c) the linear-regression sub-model, and (d) the integrated model. 
IF $y(k-1)$ is AND $y(k-2)$ is THEN $g(k)$ is
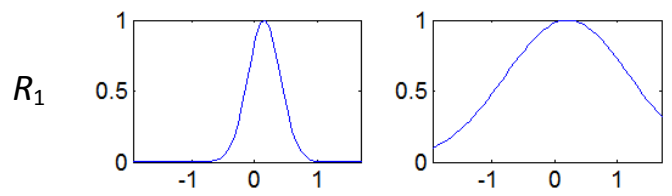

$Y_{A N N}$
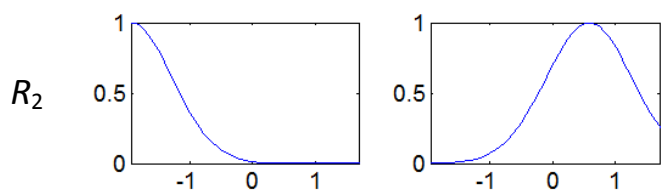

$$
Y_{A N N}
$$
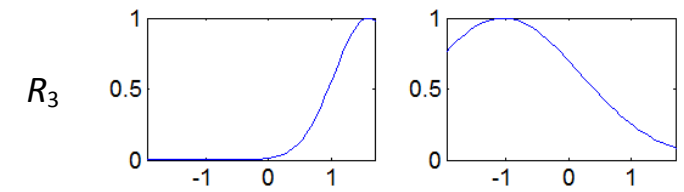

$Y_{F M}$
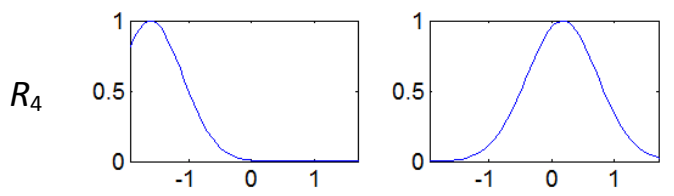

$Y_{F M}$
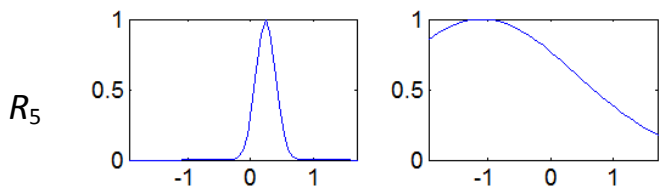

$Y_{A N N}$

Fig. 8. Master fuzzy rules for the dynamical system identification problem.
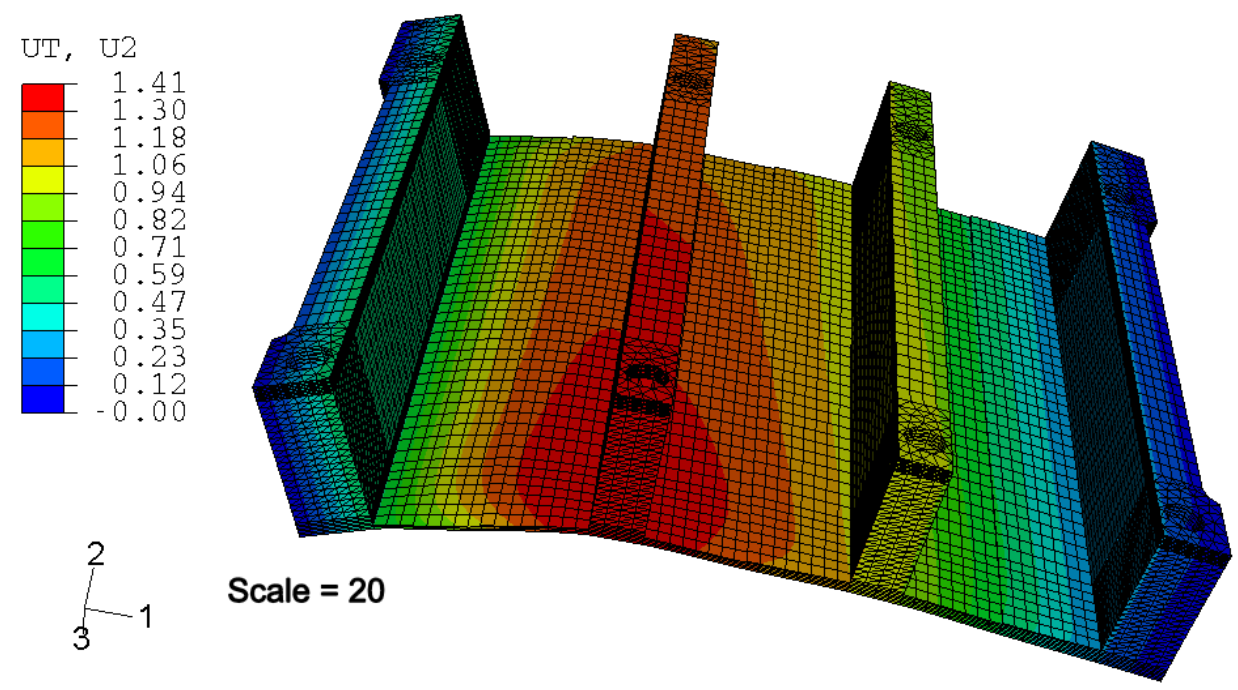

Fig. 9. Part distortion of a machined component under residual stresses [34]. 


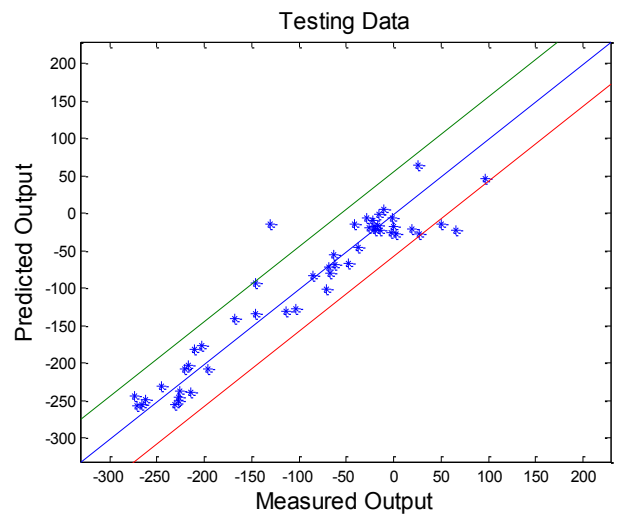

(a)

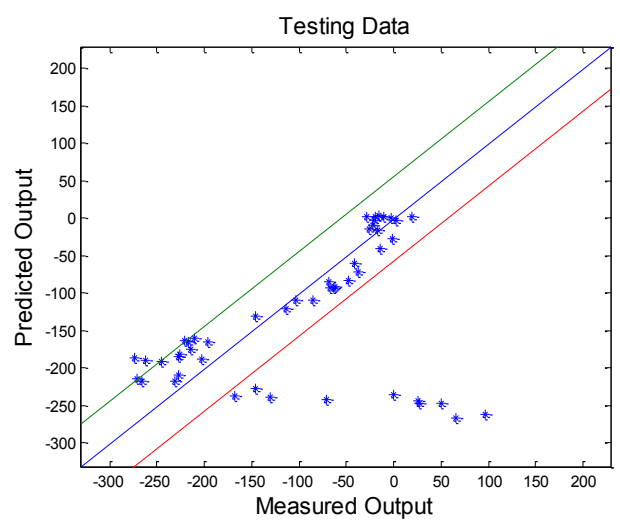

(c)

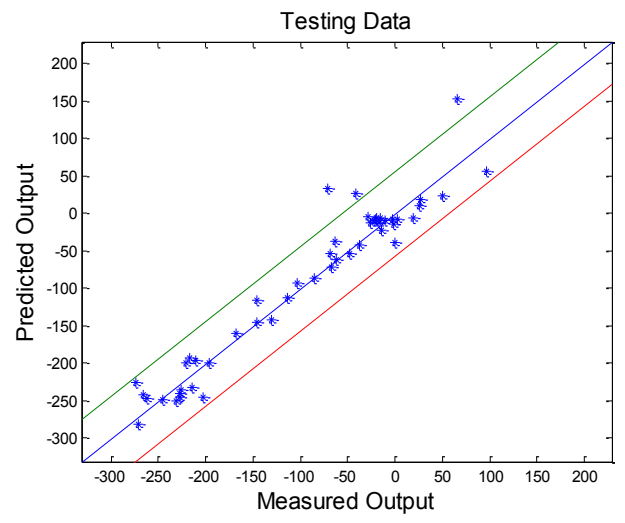

(b)

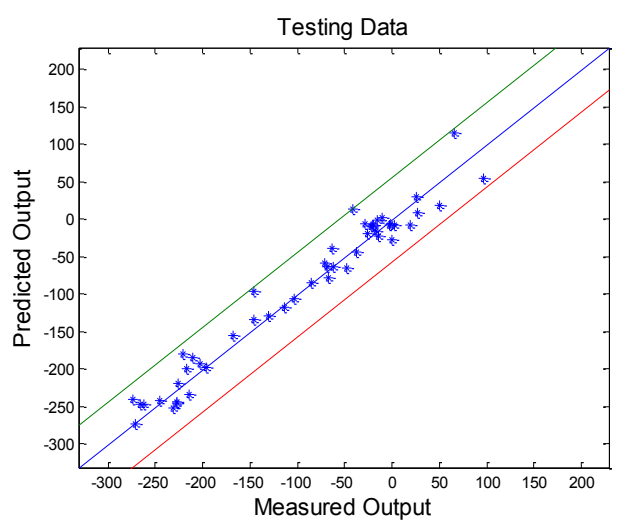

(d)

Fig. 10. The residual stress models' predicted outputs versus measured outputs based on the testing data (with the $+10 \%$ and $-10 \%$ error bands): (a) the fuzzy sub-model, (b) the neuralnetwork sub-model, (c) the linear-regression sub-model, and (d) the integrated model.

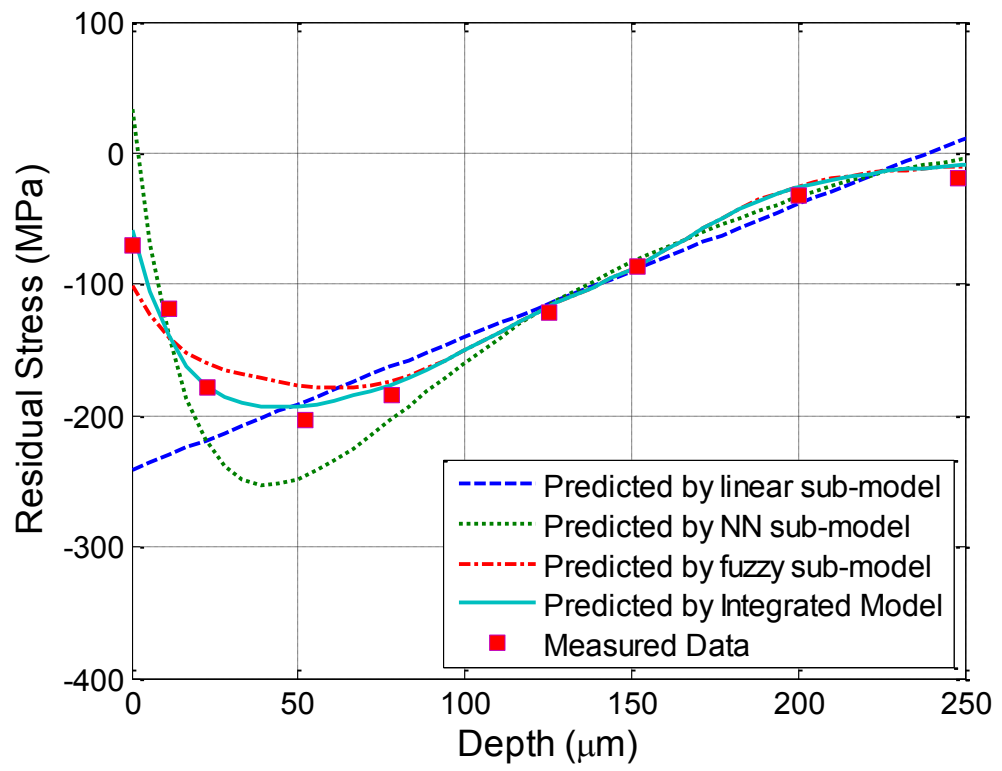

Fig. 11. The predictive residual stress curves and measured data. 
$R_{1}$

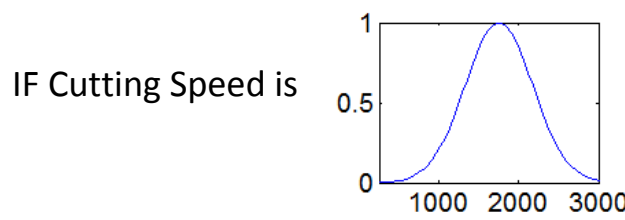

\section{AND Feed per}

Tooth is

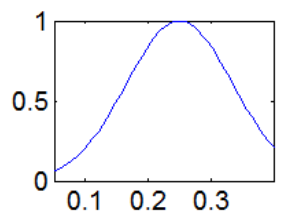

AND Feed Velocity

is

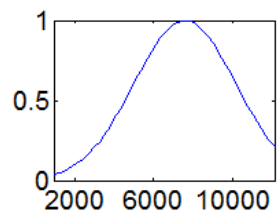

AND Coolant is
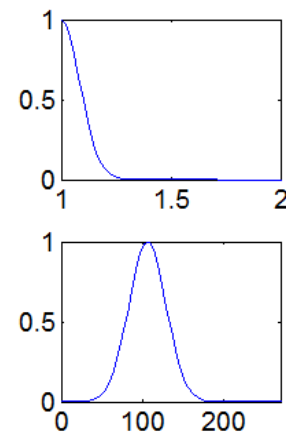

$R_{2}$
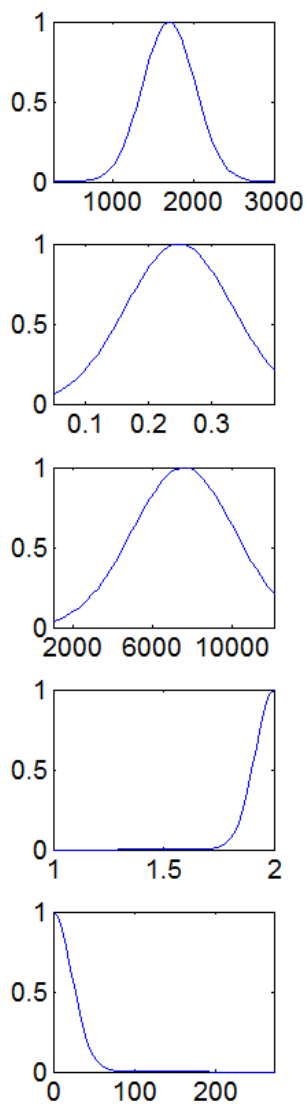

$R_{3}$
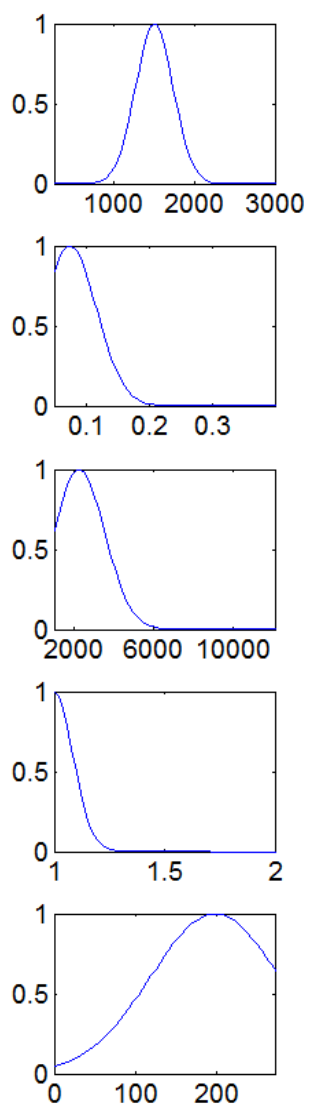

THEN Residual

Stress is

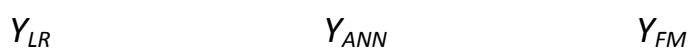

Fig. 12. Master fuzzy rules of the residual stress model. 


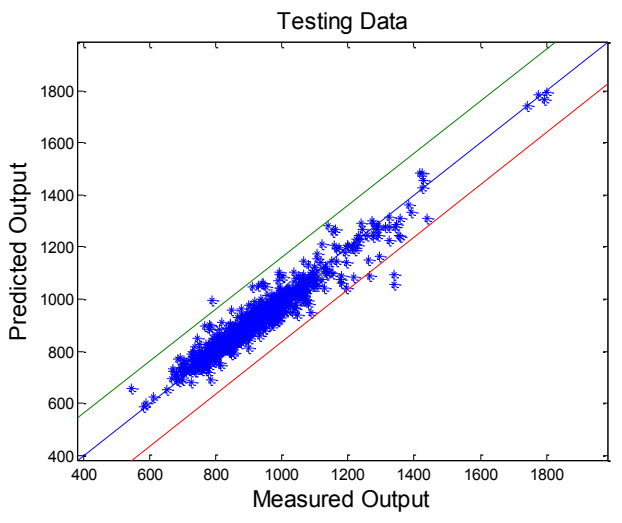

(a)

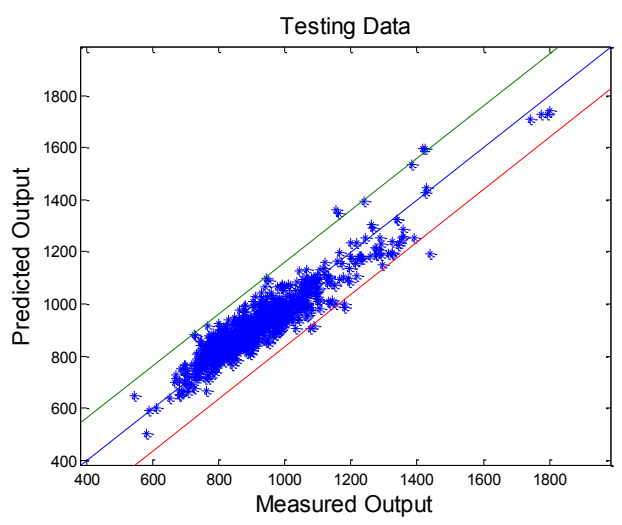

(c)

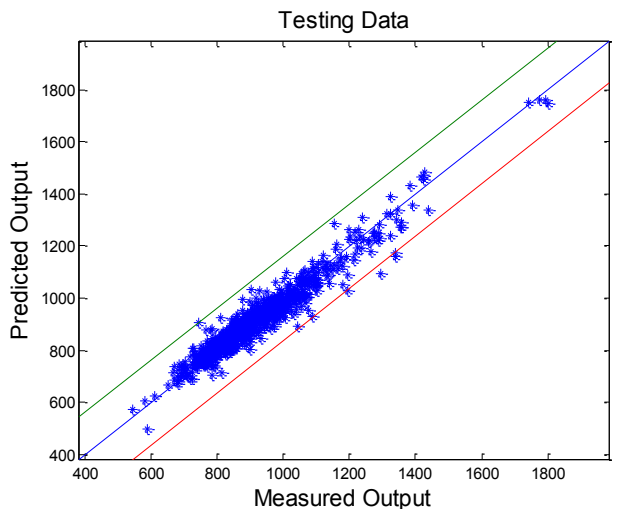

(b)

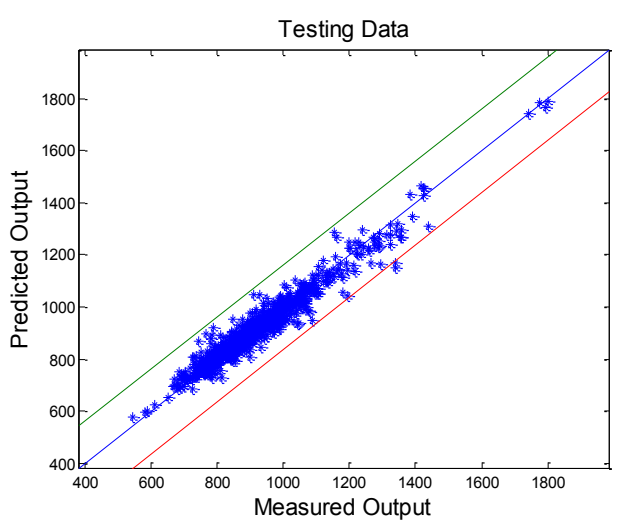

(d)

Fig. 13. The UTS models' predicted outputs versus measured outputs based on the testing data (with the $+10 \%$ and $-10 \%$ error bands): (a) the fuzzy sub-model, (b) the neural-network sub-model, (c) the linear-regression sub-model, and (d) the integrated model. 


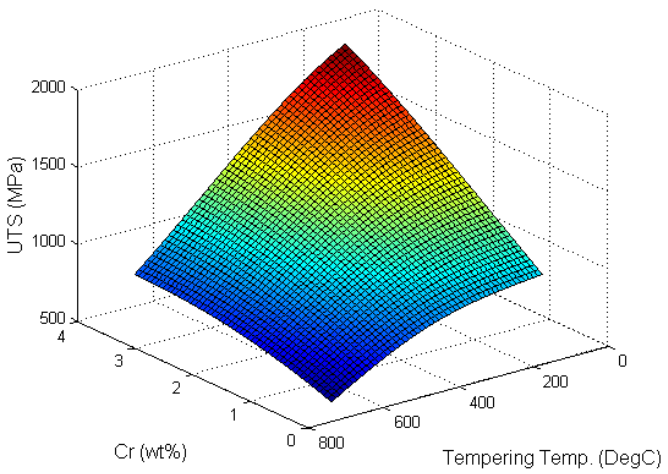

(a)

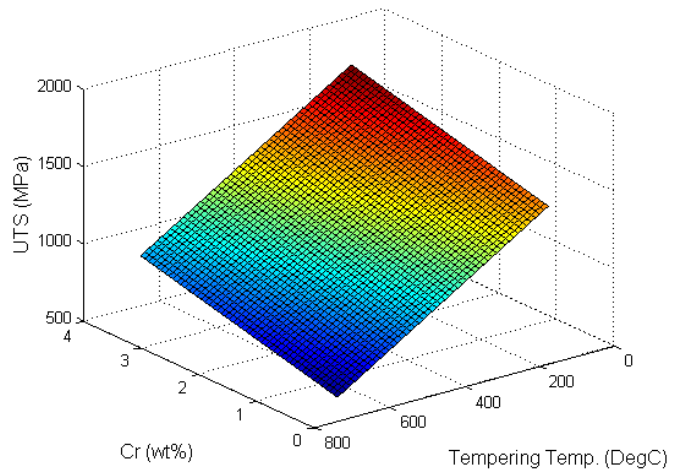

(c)

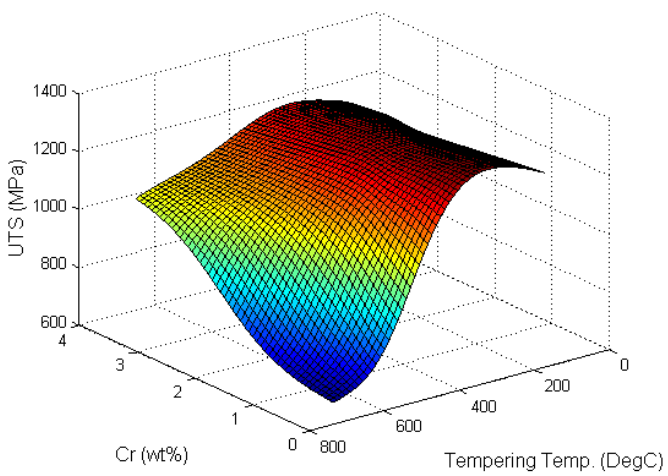

(b)

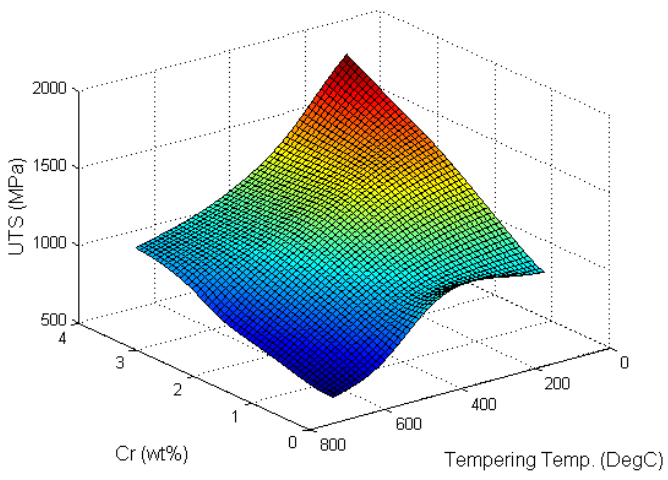

(d)

Fig. 14. The UTS models' response surfaces: (a) the fuzzy sub-model, (b) the neural-network sub-model, (c) the linear-regression sub-model, and (d) the integrated model. 


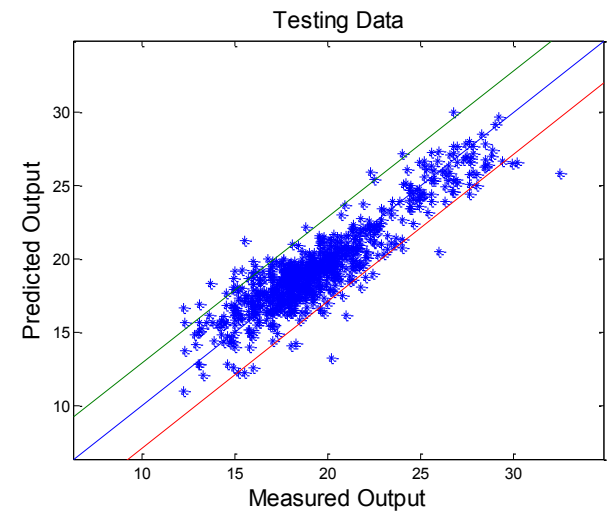

(a)

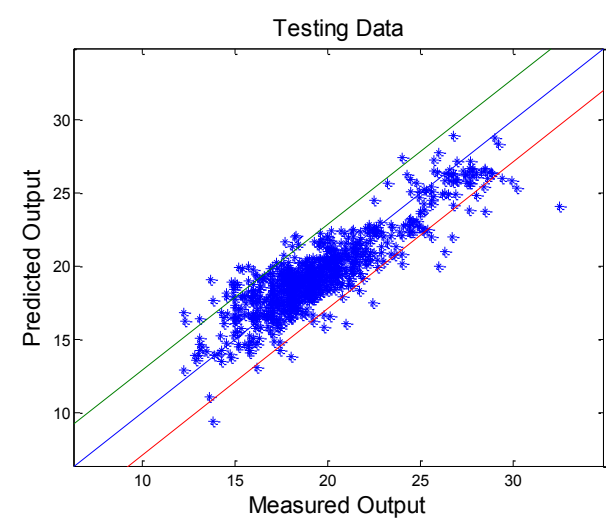

(c)

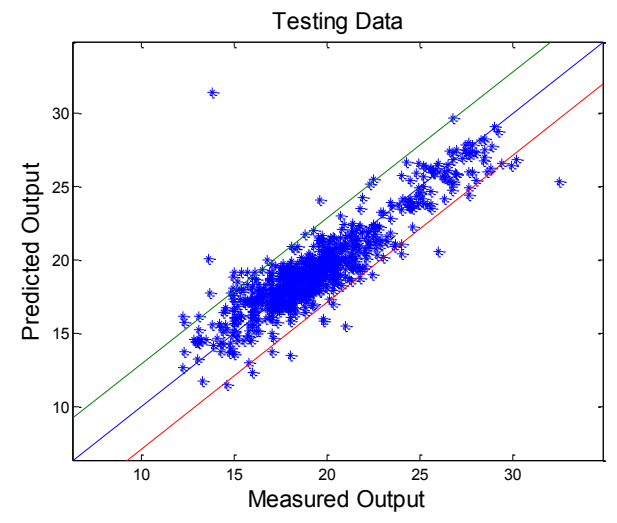

(b)

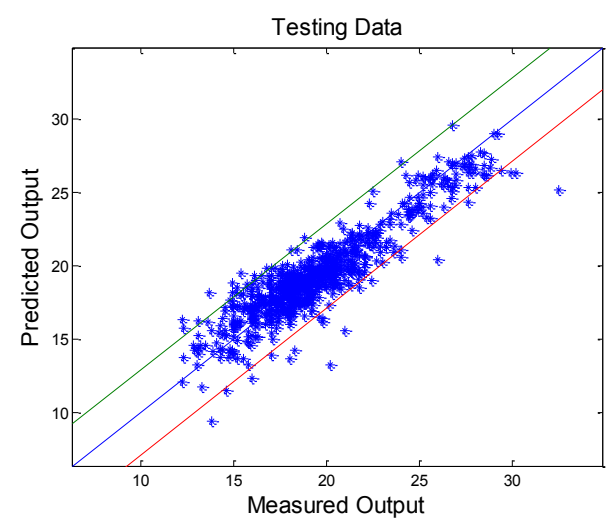

(d)

Fig. 15. The elongation models' predicted outputs versus measured outputs based on the testing data: (a) the fuzzy sub-model, (b) the neural-network sub-model, (c) the linearregression sub-model, and (d) the integrated model. 


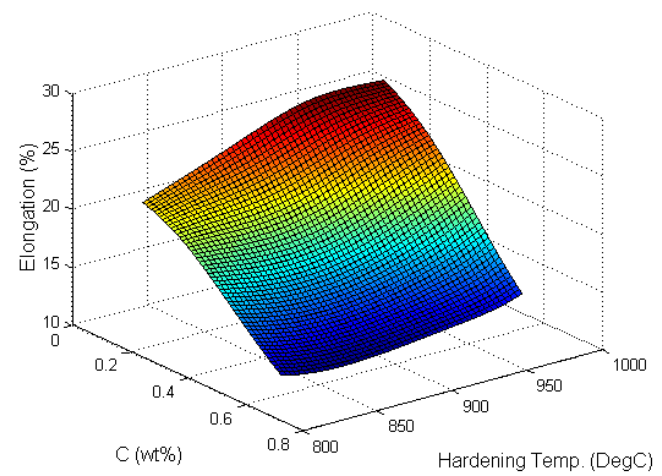

(a)

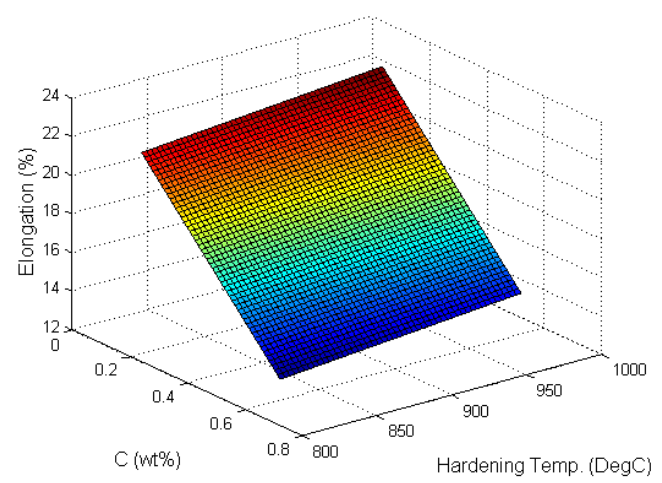

(c)

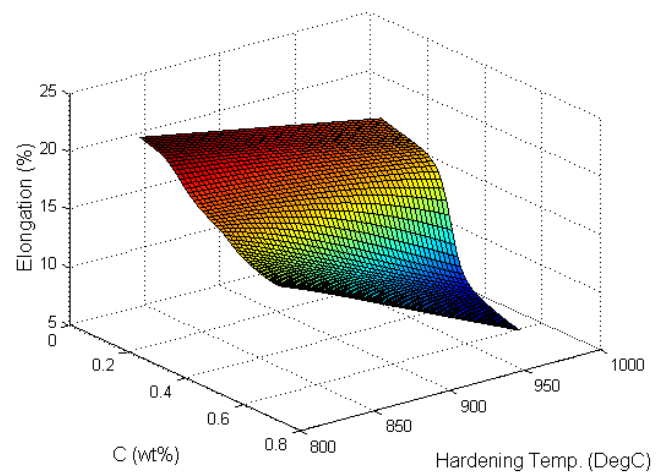

(b)

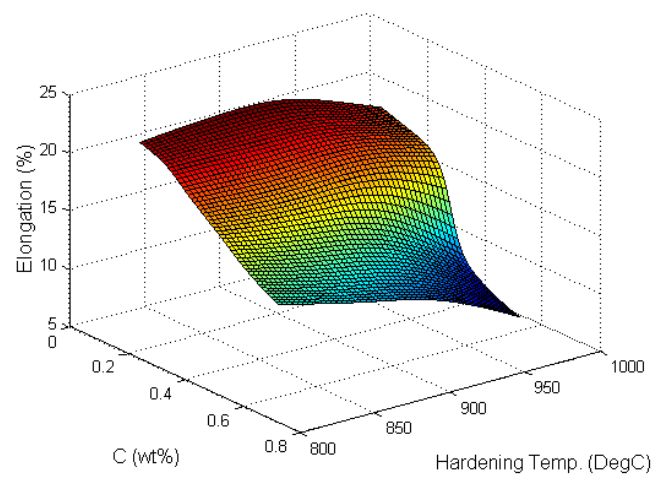

(d)

Fig. 16. The elongation models' response surfaces: (a) the fuzzy sub-model, (b) the neuralnetwork sub-model, (c) the linear-regression sub-model, and (d) the integrated model. 